\title{
No lesion in the liver can also be hepatic tuberculosis - An important consideration
}

\begin{abstract}
Hepatic tuberculosis (TB) is rare, but recently it is more frequently detected. On imaging, it presents with different appearances and can mimic a variety of other conditions. A high degree of suspicion combined with appropriate diagnostic modalities and image-guided tissue sampling examination greatly aid in the timely diagnosis of the disease. The sights of this case report are to illustrate and discuss the different presentation of hepatic TB on computed tomography. This entity is frequently related to caseous necrosis, which is the hallmark of this disease. However, our patient didn't have any liver lesion in spite of deranged liver function tests.
\end{abstract}

Keywords: ultrasound, hepatic tuberculosis, contrast-enhanced computed tomography

\author{
Volume II Issue 5 - 202 I
}

\section{Kritika, Rajaram Sharma, Tapendra Tiwari, Saurabh Goyal}

Pacific Institute of Medical Sciences, India

Correspondence: Rajaram Sharma, Assistant professor, Radiodiagnosis Pacific Institute of Medical Sciences (PIMS), India, Email hemantgalarial3@gmail.com

Received: September 17, 2021 | Published: October 07, 2021
Abbreviations: USG, ultrasound; AST, aspartate aminotransferase; ALT, alanine transaminase; CECT, contrastenhanced computed tomography

\section{Introduction}

Tuberculosis is an old entity presenting with new forms and with the help of advanced cross-sectional imagingthese are more frequently detected. The most commonly affected organ is lung but can involve any other organ, also denoted as extrapulmonary type. TB of the liver is the typical presentation and report less than $1 \%$ of all tuberculous infections. ${ }^{1}$ Its occurrence is low due to a lack of tissue oxygen tension in the liver, which is unsuitable for mycobacterial growth. The disease is seen at any age but is most commonly spotted in young adults.

\section{Case report}

A formerly healthy 43-year-old man presented to our hospital with a vague continuous type of pain in abdomen, predominantly in the right hypochondrium that started three months back. The pain used to be aggravated after meals and patient also experienced nausea and vomiting sometimes. There was a history of occasional fever for the last three months. Physical examination of the patient revealed palpable lymph nodes in bilateral cervical chains and hepatomegaly.

On initial investigations, the patient had deranged liver functions, i.e. raised aspartate aminotransferase(AST), alanine transaminase (ALT) and alkaline phosphatase levels. Among these, only alkaline phosphatase elevation raised the probability of tubercular hepatic parenchymal involvement. Further, the patient underwent an ultrasound (US) examination of the abdomen, which revealed hepatosplenomegaly and abdominal lymphadenopathy. Multiple splenic microabscesses were also noted in US examination, but no apparent large hypoechoic lesions were present in the liver parenchyma. For better delineation of the diseases, contrast-enhanced computed tomography (CECT) of the abdomen was advised.

The scan was done on 128 slice Siemens CT scan machine, which revealed moderate hepatomegaly with liver measuring $17.5 \mathrm{~cm}$ in craniocaudal dimension (Figure 1A). There were multiple conglomerated and enlarged lymph nodes with internal low attenuating areas (signify necrosis) observed in pre-para aortic, aortocaval and mesenteric regions (Figure 1B), largest measuring (20x11)mm in the right iliac fossa. The spleen was also enlarged with numerous tiny hypodense peripherally enhancing lesions diffusely scattered in its parenchyma (Figure 2). A minimal amount of free fluid was noted in the peritoneal cavity. The CT scan also demonstrated multiple centrilobular nodular densities arranged in linear branching patterns forming tree-in-bud appearance and patchy areas of consolidations in the limited scan of the bilateral lung fields (Figure 3A). Mild rightsided pleural effusions and left-sided empyema were also noted (Figure 3B).

Given persistently raised liver enzymes and without any demonstrable liver parenchymal involvement on CECT, ultrasonography (USG) guided liver biopsy was performed, revealing multiple epithelioid cell granulomas with Langhans giant cells and caseation necrosis (Figure 4). Acid-fast bacilli were also demonstrated in the specimens.

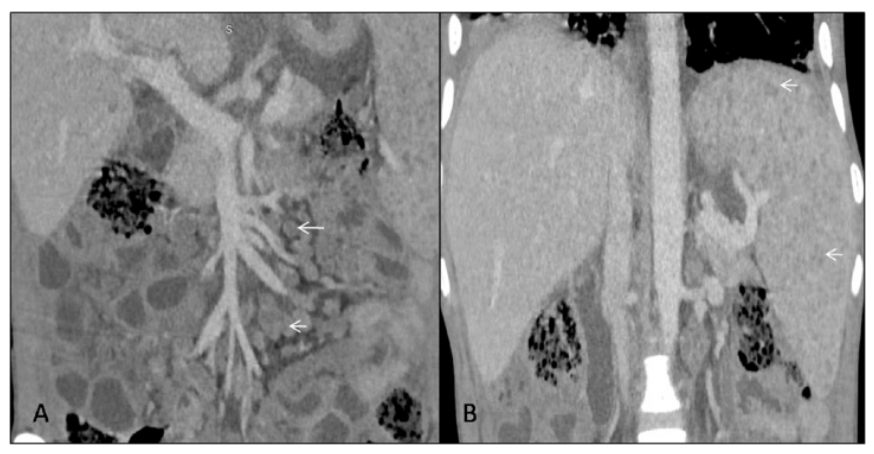

Figure I Contrast enhanced computed tomography scan, venous phase in coronal plane, (A) shows multiple conglomerated, peripherally enhancing lymph nodes with central low attenuation in mesentery adjacent to mesenteric vessels (white arrow). (B) shows mild enlargement of the liver and spleen (craniocaudal length measures approx $17 \mathrm{~cm}$ and $16 \mathrm{~cm}$ respectively). Numerous tiny hypodense peripherally enhancing lesions are seen diffusely scattered insplenicparenchyma (white arrow). 


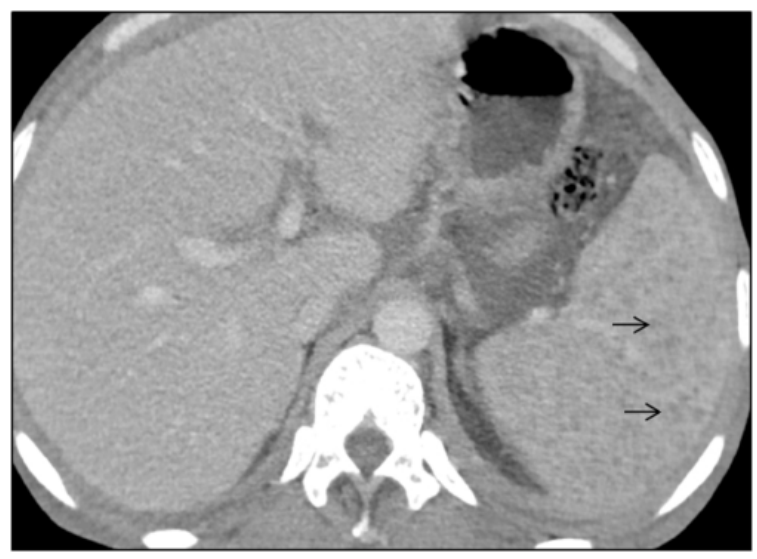

Figure 2 Contrast enhanced computed tomography scan, venous phase in axial plane shows numerous tiny hypodense peripherally enhancing lesions diffusely scattered in splenic parenchyma consistent with tubercular micro abscesses (black arrows).

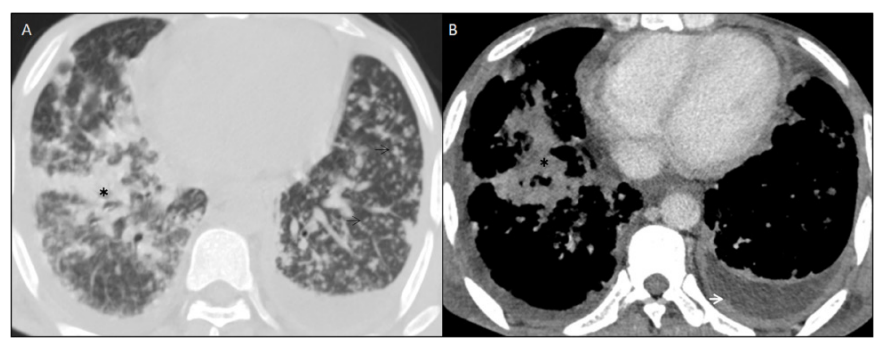

Figure 3 CECT of bilateral lower hemithorax in axial planes (A) Lung window, shows multiple tiny centrilobular nodular opacities in bilateral lung fields (black arrows) forming "tree-in-bud appearance" and patchy consolidation in right lower lobe (black asterisk) signifies active pulmonary tuberculosis. (B) Soft tissue window reveals thickened and enhancing left pleural linings with fluid collection in pleural space forming "split pleura sign" suggesting empyema.

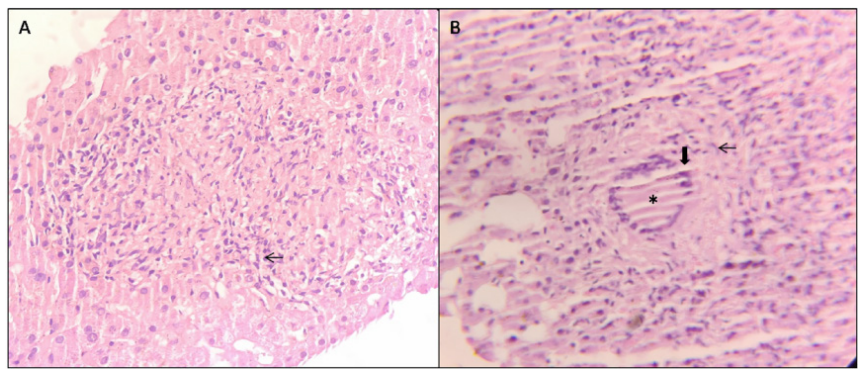

Figure 4 40X microscopic image showing elongated epitheloid cells (black arrow), granuloma with surrounding necrosis (asterisk) and lymphocytes (black thick arrow).

\section{Discussion}

Hepatic tuberculoma is a highly unusual infection of the liver. Hepatic tuberculosis is categorised as (a) primary acute pulmonary tuberculosis with liver participation, (b) primary tuberculosis of the liver, (c) miliary tuberculosis, (d) tuberculoma or abscess formation, (e) chronic pulmonary tuberculosis with liver parenchymal participation, and (f) tubercular cholangitis. ${ }^{2}$ The route of transformation of tubercular bacilli is through hematogenous dissemination. Miliary tuberculosis is usually disseminated through the arterial system, whereas in the liver, it travels through portal circulation (portal vein). Firstly, granulomas (both caseating and non-caseating granulomas types) formations occur, which are often seen in periportal areas. In focal tuberculosis, numerous granulomas coalesce to form a large tumour. Broadly, hepatic tuberculosis is categorised into three forms. The commonest one is diffuse liver involvement with pulmonary or miliary tuberculosis, which occur in $50-80 \%$ of cases. The second one is diffuse hepatic infiltration without lung involvement, termed as granulomatous hepatitis. The third or the rarest is focal/local tuberculoma or abscess. Fever is the most common presentation seen in granulomatous disease of the liver, accounting for $63-99 \%$ times followed by abdominal pain, weight loss and hepatomegaly being present in $50 \%$ of cases with splenomegaly in one third. ${ }^{3}$ The laboratory investigations may reveal anaemia with raised ESR and mild elevation of liver enzymes due to the injury to hepatic parenchyma. ${ }^{4}$ The ultrasound imaging reveals hypoechoic lesions or lesions with the hyperechoic rim. CECT portray granulomatous involvement of the liver, which appears as variable sized hypodense lesions that tend to be distinct with no pathognomonic trademark to differentiate them from other infective or some neoplastic lesions. ${ }^{5}$ MRI offers no added benefit to the CECT findings. ${ }^{6}$

In the abdomen, lymphadenopathy is the most common imaging finding of tuberculosis, being seen in $55 \%-66 \%$ of patients. ${ }^{7}$ The usual pattern of involvement is seen in the peripancreatic and mesenteric lymph node groups in the form of nodal enlargement and conglomeration. The maximum $(40 \%-60 \%)$ of affected patients with lymphadenitis have enlarged lymph nodes with low attenuating centers and high attenuating enhancing rims on CECT that are precise for this entity but not pathognomonic for caseous necrosis. ${ }^{7,8}$ These nodal enlargements may cause biliary, gastrointestinal, or genitourinary obstruction, which encourages an alternative diagnosis.

Other nodal involvement patterns described are conglomerated heterogeneously attenuated masses which forms from combining an increased number (more than three) of average or slightly enlarged homogeneous nodes.

The final diagnosis is decided based on histopathological evidence of caseating granulomas, the presence of AFB on smear or after the culture of the biopsy specimen. On tissue biopsy, epithelioid granulomas may be found in approximately $80-100 \%$ of cases and caseating necrosis in $30-83 \%$ of cases. Culture positivity for tuberculosis is seen only in $10 \%$ of granulomas. ${ }^{6}$ The mainstay of treatment is standard antitubercular therapy.

\section{Conclusion}

TB of the liver is a very rare entity, but it is essential to recognise the imaging manifestations because of its increasing detection rate and its nature to mimic many other intra-abdominal pathologies. Caseous necrosis is the key feature found in imaging of hepatic tuberculosis, which is the hallmark of this entity. However, hepatic lesions can be of various kinds, or no lesion in the liver can be the manifestation. Solo Cross-sectional imaging may be insufficient in reaching a final diagnosis. So, image-guided tissue biopsy is needed in most cases. Concurrently, other organ involvements like spleen, lung or lymph nodes are helpful features towards the diagnosis.

\section{Acknowledgments}

None.

\section{Conflicts of interest}

The authors declare no conflicts of interest. 


\section{Funding}

None.

\section{References}

1. Chong VH. Hepatobiliary tuberculosis: a review of presentations and outcomes. South Med J. 2008;101(4):356-361.

2. Vainrub B. Diagnostic procedure in the evaluation of liver disease. $L a b$ Res Methods Biol Med. 1983;7:119-112.

3. Hersh C. Tuberculosis of the liver. A study of 200 cases. S Afr Med J. 1964;38(11):857-863.
4. Gupta S, Meena HS, Chopra R. Hepatic involvement in tuberculosis. $J$ Assoc Physicians India. 1993;41:20-22.

5. Levine C. Primary macronodular hepatic tuberculosis: US and CT appearances. Gastrointes Radiol. 1990;15:307.

6. Puri AS, Nayyar AK, Vij JC. Hepatic Tuberculosis. Indian $J$ Tub. 1994;41(3):131-134.

7. Leder RA, Low VH. Tuberculosis of the abdomen. Radiol Clin North Am. 1995;33(4):691-705.

8. Denton T, Hossain J. A radiological study of abdominal tuberculosis in a Saudi population, with special reference to ultrasound and computed tomography.Clin Radiol. 1993;47(6):409 - 414. 